\title{
Universal entanglement transformations without communication
}

\author{
Wim van Dam* \\ Mathematical Sciences Research Institute, 1000 Centennial Drive \#5070, Berkeley, California 94720-5070, USA \\ Patrick Hayden \\ Institute for Quantum Information, Caltech 107-81, Pasadena, California 91125, USA
}

(Received 10 January 2002; published 11 June 2003)

\begin{abstract}
We show that in the presence of finite catalysts, any pure bipartite entangled state can be converted into any other, to unlimited accuracy, without the use of any communication, quantum or classical. We call this process embezzling entanglement because it involves removing a small amount of entanglement from the catalyst in a physically unnoticeable way.
\end{abstract}

DOI: 10.1103/PhysRevA.67.060302

PACS number(s): 03.67.Hk, 03.67.Mn

The interconvertibility of entangled quantum states is an important question in quantum-information theory, both for its own sake and because of its connections to quantum error correction [1], quantum cryptography [2], and quantum communication complexity [3]. In 1999, Nielsen and Hardy supplied a powerful tool for studying this problem, in the form of a simple characterization of the bipartite pure states convertible into each other using only local operations and classical communication (LOCC) [4,5]. Building on that work, complete characterizations of the corresponding probabilistic [6] and approximate [7] conversion problems soon followed. In addition, Jonathan and Plenio discovered the existence of catalysts: states that are recovered once a transformation is complete but whose presence allows successful LOCC protocols that would not otherwise have been possible [8].

In this paper, we exhibit a family of bipartite catalysts $\{|\mu(n)\rangle\}_{n=1}^{\infty}$ such that, for any $\varepsilon>0$ and any bipartite state $\left|\varphi_{A B}\right\rangle$, the transformation

$$
|\mu(n)\rangle \mapsto|\mu(n)\rangle \otimes\left|\varphi_{A B}\right\rangle
$$

can be accomplished with fidelity better than $1-\varepsilon$, for all sufficiently large $n$ without any communication, quantum or classical. In other words, it is possible to embezzle a copy of $\left|\varphi_{A B}\right\rangle$ from $|\mu(n)\rangle$, thereby removing a small amount of entanglement from the original state, while causing only an arbitrarily small disturbance $\varepsilon$ to it. This embezzlement protocol only requires the two parties $A$ and $B$ to rearrange the coefficients of the $\mu(n)$ state such that it resembles the desired $\mu(n) \otimes \varphi_{A B}$. (An analogy to this phenomenon is illustrated in Fig. 1.) Because the set of states $\{\mu(n)\}$ can be used to embezzle any target state $\varphi$ to within an arbitrarily high fidelity $1-\varepsilon$ that depends only on the Schmidt rank of $\varphi$ and the size $n$, we call the set a universal embezzling family. It follows trivially that this family can also be used as a catalyst to "convert" any now superfluous $\psi_{A B}$ to $\varphi_{A B}$ with arbitrarily small error.

\footnotetext{
*Also at UC Berkeley and HP Labs, Palo Alto; electronic address: vandam@cs.berkeley.edu

${ }^{\dagger}$ Electronic address: patrick@cs.caltech.edu
}

It should be noted that the embezzlement protocol does more than creating the illusion that entanglement has been increased without communication. Such an illusion is possible for every initial state $\mu$ because the Von Neumann entropy fails to be continuous in the limit of infinitedimensional systems. (Consider, for example, the fact that the state $\sqrt{1-\varepsilon}|0\rangle_{A}|0\rangle_{B}+\sqrt{(\varepsilon / n)} \sum_{j=1}^{n}|j\rangle_{A}|j\rangle_{B}$ will have fidelity $\sqrt{1-\varepsilon}$ with the classical state $|0\rangle_{A}|0\rangle_{B}$, while its entanglement will be approximately $\varepsilon \log n$ bits.) Rather, the embezzlement procedure that is presented here shows how for every specific state $\varphi$ one can transform $\mu$ into $\mu \otimes \varphi$ (approximately). This is a more specific task, which is only possible if we use a proper initial state $\mu$. Indeed, following the optimal protocol described in Ref. [7], it is easy to see that with a maximally entangled state $|\mu\rangle$ $=(1 / \sqrt{n}) \sum_{i=1}^{n}|i\rangle_{A}|i\rangle_{B}$, it is impossible to perform any nontrivial embezzlement.

The index $n$ indicates the Schmidt rank of the specific $|\mu(n)\rangle$, and for each $n$ the embezzling state is defined by

$$
|\mu(n)\rangle:=\frac{1}{\sqrt{C(n)}} \sum_{j=1}^{n} \frac{1}{\sqrt{j}}|j\rangle_{A}|j\rangle_{B},
$$

where $C(n):=\sum_{j=1}^{n}(1 / j)$ is chosen so that $|\mu(n)\rangle$ is normalized. Now suppose, we would like to embezzle the state $\left|\varphi_{A B}\right\rangle:=\sum_{i=1}^{m} \alpha_{i}|i\rangle_{A}|i\rangle_{B}$ from $|\mu(n)\rangle$, where $\left|\varphi_{A B}\right\rangle$ is written according to its Schmidt decomposition such that all $\alpha_{i}$ amplitudes are positive reals. This problem is equivalent to creating the state $|\omega(n)\rangle=\sum_{j=1}^{m n} \omega_{j}|j\rangle_{A}|j\rangle_{B}$, which is defined as the state with the same Schmidt basis and coefficients as $|\mu(n)\rangle \otimes\left|\varphi_{A B}\right\rangle$ but with the coefficients $\omega_{j}$ in decreasing order. Thus, $|\omega(n)\rangle$ can be converted into $|\mu(n)\rangle \otimes\left|\varphi_{A B}\right\rangle$ exactly by local unitary operations alone. The embezzlement protocol will simply consist of performing these local unitaries because we will show that $|\langle\mu(n) \mid \omega(n)\rangle|$ goes to 1 as $n$ goes to infinity.

The first step in the proof will be to show that the first $n$ Schmidt coefficients of $|\omega(n)\rangle$ are smaller than the corresponding ones of $|\mu(n)\rangle$. To see this, observe that these first $n$ Schmidt coefficients of $|\omega(n)\rangle$ are all of the form $\alpha_{i} / \sqrt{j C(n)}$, where $1 \leqslant i \leqslant m$ and $1 \leqslant j \leqslant n$. For a fixed $t$ and $i$, we let $N_{i}^{t}$ be the number of such coefficients $\alpha_{i} / \sqrt{j C(n)}$ 


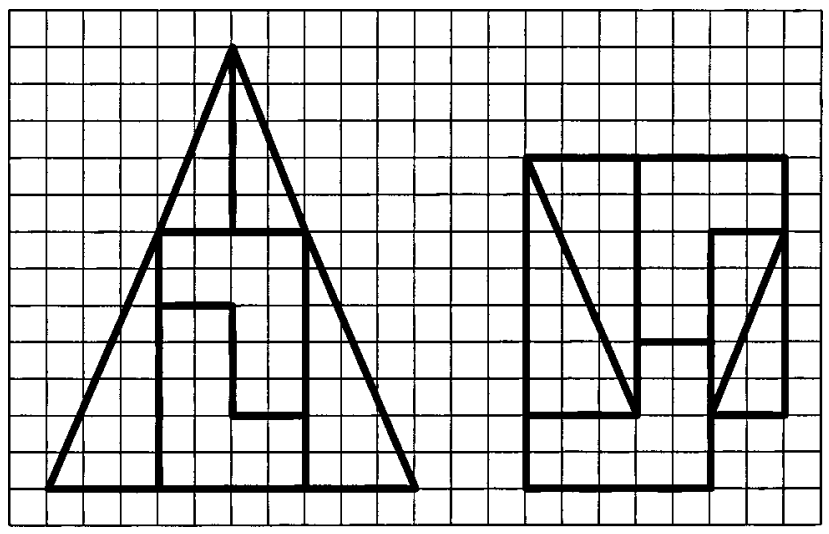

FIG. 1. An illustration of the "embezzlement effect." By a wellchosen rearrangement, we can create the suggestion that the six pieces of the rightmost figure, with area size 59, can also be used to cover the triangle on the left with its surface of 60 units. A similar phenomenon is described in this paper for the entanglement of a distributed quantum state. It is shown, how we can reorder the amplitudes of an embezzling state $\mu$ such that we get a very close approximation of an enlarged state $\mu \otimes \varphi$, which appears to have significantly more entanglement than the original $\mu$.

that are strictly greater than $1 / \sqrt{t C(n)}$. By the constraint 1 $\leqslant j<\alpha_{i}^{2} t$, it follows that $N_{i}^{t}<\alpha_{i}^{2} t$ and, since $\sum_{i=1}^{m} \alpha_{i}^{2}=1$, we can conclude that $\sum_{i=1}^{m} N_{i}^{t}<t$. This upper bound on the number of $\omega_{j}$ coefficients that are strictly bigger than $1 / \sqrt{t C(n)}$ combined with the ordering $\omega_{1} \geqslant \omega_{2} \geqslant \cdots \geqslant \omega_{m n}$ proves that $\omega_{j} \leqslant 1 / \sqrt{j C(n)}$ for all $1 \leqslant j \leqslant n$. Consequently, the fidelity between $|\mu(n)\rangle$ and $|\omega(n)\rangle$ can be bounded from below by

$$
|\langle\mu(n) \mid \omega(n)\rangle|=\sum_{j=1}^{n} \frac{\omega_{j}}{\sqrt{j C(n)}} \geqslant \sum_{j=1}^{n} \omega_{j}^{2} .
$$

Our next task is to show that this sum is close to 1 for large $n$. Let $|\psi(n)\rangle:=|\mu(n)\rangle \otimes\left|\Phi^{m}\right\rangle$, where $\left|\Phi^{m}\right\rangle$ $:=(1 / \sqrt{m}) \sum_{i=1}^{m}|i\rangle_{A}|i\rangle_{B}$ is the maximally entangled state of rank $m$. Then $\omega(d)_{A}>\psi(d)_{A}$ and it follows that $\sum_{j=1}^{n} \omega_{j}^{2}$ $\geqslant \sum_{j=1}^{n} \beta_{j}^{2}$, where $\left(\beta_{j}\right)$ is the vector of Schmidt coefficients of $|\psi(n)\rangle$ in decreasing order. This last sum is easy to evaluate, however,

$$
\sum_{j=1}^{n} \beta_{j}^{2} \geqslant \sum_{j=1}^{\lfloor n / m\rfloor} \sum_{i=1}^{m} \frac{1}{j C(n) m} \geqslant 1-\frac{\log (m)}{\log (n)}
$$

Thus, for any fidelity $1-\varepsilon<1$, the requirement $n>m^{(1 / \varepsilon)}$ on $|\mu(n)\rangle$ suffices. If we view the state $\left|\varphi_{A B}\right\rangle$ as a string of $\log m$ pairs of qubits then $|\mu(n)\rangle$ need only consist of $(1 / \varepsilon) \log m$ pairs of qubits, which is only linear in the number of qubits of $\left|\varphi_{A B}\right\rangle$.

The embezzlement protocol we present here requires absolutely no communication and uses the same set of catalysts for every input. Is it possible that by tailoring the catalyst to the target state as well as making use of local operations and classical communication that we could find more effective embezzlement schemes? Not significantly. Let $n$ be the Schmidt rank of the catalyst $|\xi\rangle$ and consider the transforma- tion $|\xi\rangle \mapsto|\xi\rangle \otimes\left|\varphi_{A B}\right\rangle$. Suppose the optimal LOCC protocol yields the state $\sigma_{A B}$. In Ref. [7], it was shown that this optimal $\sigma_{A B}$ will be a pure state with Schmidt basis matching that of $|\xi\rangle \otimes\left|\varphi_{A B}\right\rangle$. Since the entanglement cannot be increased by an LOCC protocol, $S\left(\sigma_{A}\right) \leqslant S\left(\xi_{A}\right)$. Therefore, if $\operatorname{Tr}\left|\sigma_{A}-\xi_{A} \otimes \varphi_{A}\right|=\delta$, the Fannes' inequality [9] implies that, for $\delta<1 / e$,

$$
S\left(\varphi_{A}\right) \leqslant\left|S\left(\xi_{A} \otimes \varphi_{A}\right)-S\left(\sigma_{A}\right)\right|<\delta(\log (m)+\log (n))+\eta(\delta),
$$

where $\eta(\delta)=-\delta \log \delta$ and $m$ is the rank of $\varphi_{A}$, and hence

$$
\frac{S\left(\varphi_{A}\right)-\eta(\delta)}{\log (m)+\log (n)}<\delta
$$

For our protocol, however, a straightforward calculation reveals that

$$
\begin{aligned}
\delta & =\operatorname{Tr}\left|\omega(n)_{A}-\mu(n)_{A}\right|=\sum_{j=1}^{n}\left(\mu_{j}^{2}-\omega_{j}^{2}\right)+\sum_{j=n+1}^{n m} \omega_{j}^{2} \\
& \leqslant \frac{2 \log (m)}{\log (n)}
\end{aligned}
$$

where we used the fact that $\mu_{j} \geqslant \omega_{j}$ for $1 \leqslant j \leqslant n$ and $\mu_{j}=0$ for $j>n$ combined with the bound of Eq. (4). Clearly, for a fixed $\varphi_{A B}$ this $\delta$ saturates Eq. (6) to within a constant factor for large $n$.

We have shown that it possible to embezzle entanglement without any communication whatsoever and that the set $\{|\mu(n)\rangle\}$ can be used to embezzle any bipartite pure state. Furthermore, we have shown that the universal family $\{|\mu(n)\rangle\}$ is nearly optimal, almost saturating the limit on embezzlement imposed by the continuity of the Von Neumann entropy.

The embezzlement phenomenon has a number of consequences for the study of quantum information. For example, it implies that the trumping relation on bipartite entangled states [10] is not stable to arbitrarily small perturbations. In other words, in the presence of unrestricted catalysts, all states are effectively reachable from all others without communication. Similarly, a standard proof technique in quantum communication complexity reduces distributed function evaluations to related state transformations [11]. The amount of communication for the distributed problem is related to the amount of communication required to perform the corresponding state transformation. Our results imply that this technique will fail on attempts to study the probabilistic communication complexity of functions, when an unlimited amount of initial entanglement is allowed.

We would like to thank Sumit Daftuar for helpful conversations. This work was supported in part by the National Science Foundation under Grant No. EIA-0086038 and the Defense Advanced Research Projects Agency (DARPA) and Air Force Laboratory, Air Force Materiel Command, USAF, under Grant No. F30602-01-2-0524. 
[1] C.H. Bennett, D.P. DiVincenzo, J.A. Smolin, and W.K. Wootters, Phys. Rev. A 54, 3824 (1996).

[2] A.K. Ekert, Phys. Rev. Lett. 67, 661 (1991).

[3] A. Ambainis, L.J. Schulman, A. Ta-Shma, U.V. Vazirani, and A. Wigderson, in 39th Annual Symposium on Foundations of Computer Science (IEEE, Palo Alto, 1998), pp. 342.

[4] L. Hardy, Phys. Rev. A 60, 1912 (1999).

[5] M.A. Nielsen, Phys. Rev. Lett. 83, 436 (1999).

[6] G. Vidal, Phys. Rev. Lett. 83, 1046 (1999).
[7] G. Vidal, D. Jonathan, and M.A. Nielsen, Phys. Rev. A 62, 012304 (2000).

[8] D. Jonathan and M.B. Plenio, Phys. Rev. Lett. 83, 3566 (1999).

[9] M. Fannes, Commun. Math. Phys. 31, 291 (1973).

[10] S. Daftuar and M. Klimesh, e-print quant-ph/0104058.

[11] R. Cleve, W. van Dam, M. Nielsen, and A. Tapp, Lect. Notes Comput. Sci. 1509, 61 (1999). 